\title{
Maternal History and Uterine Artery Doppler in the Assessment of Risk for Development of Early- and Late-Onset Preeclampsia and Intrauterine Growth Restriction
}

\author{
Elisa Llurba, ${ }^{1}$ Elena Carreras, ${ }^{1}$ Eduard Gratacós, ${ }^{1}$ Miquel Juan, ${ }^{2}$ Judith Astor, ${ }^{3}$ \\ Angels Vives, ${ }^{4}$ Eduard Hermosilla, ${ }^{5}$ Ines Calero, ${ }^{1}$ Pilar Millán, ${ }^{1}$ \\ Bárbara García-Valdecasas, ${ }^{1}$ and Lluís Cabero ${ }^{1}$ \\ ${ }^{1}$ Department of Obstetrics, Fetal Medicine Unit, Vall d'Hebron Hospital, Universitat Autònoma de Barcelona, 08035-Barcelona, Spain \\ ${ }^{2}$ Department of Obstetrics, Son Llatze Hospital, Mallorca, Spain \\ ${ }^{3}$ Department of Obstetrics, Granoller Hospital, Barcelona, Spain \\ ${ }^{4}$ Department of Obstetrics, Terrassa Hospital, Barcelona, Spain \\ ${ }^{5}$ Department of Stadistics, Vall d'Hebron Hospital, Universitat Autònoma de Barcelona, 08035-Barcelona, Spain
}

Correspondence should be addressed to Elisa Llurba, ellurba@vhebron.net

Received 29 September 2008; Revised 27 February 2009; Accepted 12 March 2009

Recommended by Robert L. Coleman

\begin{abstract}
Objective. To examine the value of one-step uterine artery Doppler at 20 weeks of gestation in the prediction pre-eclampsia (PE) and/or intrauterine growth restriction (IUGR). Methods. A prospective multicentre study that included all women with singleton pregnancies at 19-22 weeks of gestation (w). The mean pulsatility index (mPI) of both uterine arteries was calculated. Receiveroperating characteristics curves (ROC) were drawn to compare uterine artery Doppler and maternal risk factors for the prediction of early-onset PE and/or IUGR (before $32 \mathrm{w}$ ) and late-onset PE and/or IUGR. Results. 6,586 women were included in the study. Complete outcome data was recorded for 6,035 of these women (91.6\%). PE developed in 75 (1.2\%) and IUGR in 69 (1.1\%) cases. Uterine Doppler mPI was 0.99 and the 90 th centile was 1.40 . For $10 \%$ false-positive rate, uterine Doppler mPI identified $70.6 \%$ of pregnancies that subsequently developed early-onset PE and $73.3 \%$ of pregnancies that developed early-onset IUGR. The test had a lower detection rate for the late-onset forms of the disease (23.5\% for PE and 30\% for IUGR). Maternal history has a low sensitivity in the detection of early-onset cases, although it is better at detecting late-onset PE. Conclusion. Uterine artery Doppler and maternal risk factors seem to select two different populations - early and late-onset PE which might suggest a different pathogenesis.
\end{abstract}

Copyright () 2009 Elisa Llurba et al. This is an open access article distributed under the Creative Commons Attribution License, which permits unrestricted use, distribution, and reproduction in any medium, provided the original work is properly cited.

\section{Introduction}

Pre-eclampsia (PE) and intrauterine growth restriction (IUGR), which affect $4-10 \%$ of all pregnancies, are the leading cause of premature iatrogenic deliveries and maternal morbidity in developed countries [1]. One of the major goals of fetal-maternal medicine is to detect those cases at risk early enough in pregnancy to be able to tailor interventions to improve maternal and fetal outcomes.

Both PE and IUGR are associated with pathogenic evidence of placental underperfusion and ischemia [2,3]. Blood flow through the uteroplacental circulation can be studied noninvasively using Doppler ultrasound. In pregnancies with PE or IUGR, impedance of flow in the uterine arteries increases before the clinical signs of the disease are seen $[4,5]$. These findings have been supported by histologic studies that show that Doppler resistance index is inversely related to the percentage of vessels with trophoblastic invasion [6].

Implementation of uterine Doppler as a screening test has been limited due to the high variability in the sensitivity of $\mathrm{PE}$, ranging from 30 to 80 , together with a high falsepositive rate [7]. The disparity of results when assessing efficacy of this screening test is due to differences in Doppler technique, the definition of abnormal flow velocity 
wave form, the population selected, or gestational age at the moment of screening [8]. In fact, the importance of distinguishing PE from transient hypertension in pregnancy or IUGR from constitutional small babies has only recently been considered [9]. Some studies used a two-stage program assessment [10-13], whereas nowadays one step evaluation at the moment of the anomaly scan (around $20 \mathrm{w}$ in the majority of European countries) would be enough in clinical practice [14]. There are only two studies that evaluate uterine artery Doppler screening at $20 \mathrm{w}$ of gestation $[15,16]$; however, in both cases the number of patients was too small to draw meaningful conclusions.

Therefore, the aim of our study was to examine the value of one-step uterine artery Doppler and maternal risk factors at 20 weeks of gestation in the prediction of early-onset and late-onset pre-eclampsia (PE) and/or intrauterine growth restriction (IUGR) in an unselected population. Moreover, we wanted to ascertain whether uterine artery Doppler is more effective in a high risk population.

\section{Material and Methods}

2.1. Study Population. This was a multicentric screening study involving Doppler ultrasound examination of the uterine arteries at 19-22 weeks of gestation in women with singleton pregnancies attending a routine second trimester anomaly scan. The study period was from June 2002 to May 2006.

The study was approved by the ethical committees of each hospital that took part. The participating hospitals were Hospital Son Llatzer de Mallorca, Hospital de Terrasa, Hospital de Granollers, and Hospital Vall d'Hebron de Barcelona (Spain). In these centers, all women who attended routine care were offered a transabdominal ultrasound examination at 19-22 weeks of gestation for measurement of fetal growth and examination for fetal abnormalities. All women with no major fetal abnormality were offered the option of uterine artery Doppler evaluation. Written consent was obtained in all cases. A first trimester scan was preformed in all patients, and CRL measurement was used to date the pregnancy. All sonographers were obstetricians specialized in fetal ultrasound. Quality control of screening, handling of data, and verification of adherence to protocols at the different centers were performed by the trial coordinators. Managing clinicians were blinded to the uterine Doppler measurements. As a result, there was no special follow-up for pregnant women with increased resistance in uterine arteries.

2.2. Uterine Artery Doppler Evaluation. Uterine artery Doppler velocimetry was evaluated at 19-22 weeks of gestation by abdominal ultrasound using 6-4 $\mathrm{MHz}$ probes Siemens Sonoline Antares (Siemens Medical, Erlangen, Germany); Acuson SP-10 (Acuson, Mountain View, Ca, USA); Aloka 5000 and Aloka 1700 (aloka, Tokyo, Japan); Toshiba SSH-140 (Toshiba, Tokyo, Japan).

Flow velocity waveforms of the right and left uterine arteries were imaged with the patient semirecumbent, and the uterine artery was identified on a longitudinal scan, lateral to the uterus. In that position the scan showed the bifurcation of the common iliac artery. Recording was made at the point where the uterine artery and the external iliac artery appeared to have crossed each other, as detected by color flow Doppler. Pulsed wave Doppler was then used to obtain three consecutive waveforms. Following this, pulsatility index (PI) was measured, and the presence or absence of an early diastolic notch was noted. The process was then repeated for the contralateral uterine artery, and the mean PI (mPI) of the two vessels was calculated.

Maternal history risk factors were obtained prospectively. Patients were asked to complete a questionnaire on maternal age, race, height, weight, smoking status, obstetric history (previous PE, IUGR, abruption, or stillbirth), and medical history including chronic hypertension or diabetes. Maternal history risk factors were obtained from a questionnaire, although data were confirmed, when possible, by the examination of clinical history. Demographic characteristics and Doppler findings were recorded in a computer database at the time of Doppler studies in every participating center. Data on pregnancy outcomes were obtained from examination of each patient's clinical history and labor ward records.

For the purpose of this study both IUGR and PE were classified as early onset (gestational age under 32 weeks at delivery) or late onset (32 weeks or over). The classification of pre-eclampsia at or near to term has clinical importance since early-onset PE is commonly associated with IUGR, abnormal uterine and umbilical artery Doppler evaluation and adverse maternal and neonatal outcomes. In contrast to this, late-onset PE is mainly associated with a mild maternal disease and low rate of fetal involvement, and therefore perinatal outcome is usually favorable. Patients with severe early-onset PE have different risk factors compared to late-onset $\mathrm{PE}[17,18]$. Criteria for the definition of $\mathrm{PE}$ were those of the International Society for the Study of Hypertension in Pregnancy [19]. PE was diagnosed if a previously normotensive woman had her diastolic blood pressure above $90 \mathrm{mmHg}$ measured twice (4 hours apart) and also had proteinuria of more than $300 \mathrm{mg}$ in a 24-hour urine specimen or $2+$ protein dipsticks twice ( 4 hours apart) after the 20th week of gestation. IUGR was diagnosed if the estimated fetal weight was below the 10th percentile for gestational age in our population, together with a Doppler PI in the umbilical artery above the 95th percentile, or if the estimated fetal weight was below the 3 rd percentile irrespective of the umbilical artery Doppler [20].

2.3. Statistical Analysis. Mean PI was not normally distributed and therefore expressed as median \pm interquartile range (IQR). Fisher's exact test was used to analyze maternal history variables, and independent $t$-test-Mann-Whitney $U$ test-was used for continuous variables analysis where appropiate.

The sensitivity (S), specificity (E), positive predictive value (PPV), negative predictive value (NPV), and likelihood ratio (LR) for a cut-off mean PI of 1.66 (95th centile) and for a mean PI of 1.40 (90th centile) and presence of bilateral notches in the prediction of preeclampsia and/or IUGR were 
TABLE 1: ORs for risk factors for the development of PE in the maternal history.

\begin{tabular}{|c|c|c|c|c|c|}
\hline Characteristic & & $N(\%)$ & OR & CI 95\% & $P$ \\
\hline Age & $>34$ & $17(1.5)$ & 1.33 & $(0.77-2.30)$ & .322 \\
\hline Nulliparus & & $46(1.9)$ & 2.52 & $(1.53-4.14)$ & $<.001$ \\
\hline Race other than white & & $13(1.6)$ & 1.32 & $(0.72-2.41)$ & .386 \\
\hline BMI & $\geq 30$ & $17(2.4)$ & 2.39 & $(1.37-4.15)$ & .002 \\
\hline Smoker & Yes & $7(0.5)$ & 0.33 & $(0.15-0.72)$ & .001 \\
\hline Chronic hypertension & Yes & $10(10.9)$ & 9.22 & $(4.60-18.5)$ & $<.001$ \\
\hline Diabetes I/II & Yes & $4(4.5)$ & 4.5 & $(1.38-10.8)$ & $<.031$ \\
\hline Previous PE & Yes & $9(11.1)$ & 11.22 & $(5.38-23.4)$ & $<.001$ \\
\hline Previous IUGR & Yes & $3(4.5)$ & 3.91 & $(1.2-12.7)$ & .024 \\
\hline Previous abruption & Yes & $1(4.2)$ & 3.5 & $(1.4+10.9)$ & .306 \\
\hline Previous stillbirth & Yes & $4(3.6)$ & 3.06 & $(1.1-8.55)$ & .032 \\
\hline
\end{tabular}

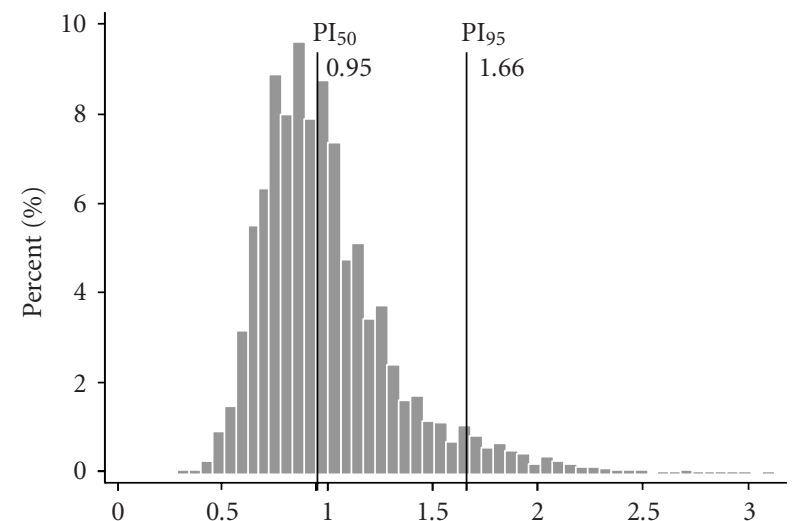

Figure 1: Frequency distribution of mean Pulsatility Index (mPI) uterine artery Doppler in the study population.

calculated. Differences were considered significant when $P<$ .05 .

Logistic regression was used to obtain the odds ratio (OR) and 95\% CI for PE in relation to maternal history variables. A multivariate analysis to draw the receiveroperating characteristics curves (ROC) was performed using maternal history variables found to be independent in a univariate analysis.

The sensitivity and specificity for different cut-offs in marker levels were calculated, and ROC were drawn to compare the performances of uterine Doppler and maternal risk factor tests. ROC analysis was also used to compare uterine artery Doppler in low- and high-risk populations.

Data was analyzed using SPSS 13.0 (SPSS, Chicago, Ill, USA) and STATA/SE 8.2 statistical packages.

\section{Results}

Doppler examination of the uterine arteries was attempted in 6586 consecutive singleton pregnancies. Satisfactory waveforms were obtained from both vessels in 6535 cases (99\%). Complete outcome data was recorded in 6035 cases $(91.6 \%)$, which formed the population for our study. No differences were found in the demographic and screening characteristics of the 6035 pregnancies with follow-up and those lost for follow-up, except for their smoking status, that was significantly higher in the group with complete follow-up (data not shown). In Table 1 we presented the maternal risk factors for the development of PE. There was an increased risk for PE in nulliparus, in noncaucasian women, in women with a body mass index (BMI) $>30$ and in those with a history of hypertension, previous PE, stillbirth, or abruption. The risk of PE decreased in cigarette smokers.

Uterine artery mPI was not normally distributed but was found to be skewed to the right with a mean value of 0.99 and the 95th percentile of 1.66 (Figure 1). In the group of women who subsequently developed PE/IUGR, mPI was higher than in those who did not develop any complication (median 1.36 $(0.70)$ versus $0.93(0.30), P<.001)$. The highest values of $\mathrm{mPI}$ were among patients that developed early-onset disease (median $1.68(0.40)$ versus $1.31(0.43), P<.01)$.

Overall, PE occurred in 75 cases (1.2\%), early-onset PE in 20 cases $(0.3 \%)$, IUGR in 69 cases $(1.1 \%)$; early-onset IUGR in 33 cases $(0.5 \%)$ and early-onset PE with IUGR in 38 cases $(0.6 \%)$. Screening characteristics for early-onset PE/IUGR between uterine artery $\mathrm{mPI}>1.66$ (95th percentile) and/or bilateral notches are set out in Table 2. Uterine artery mPI > 1.40 (90th percentile) was able to detect $73.7 \%$ of early-onset PE/IUGR with the same false-positive rate $(10 \%)$ as $\mathrm{mPI}>$ 95th plus bilateral notches, as shown in Table 3 .

In order to perform a combined assessment, uterine artery $\mathrm{mPI}$ was compared with the maternal history variables in women who subsequently developed PE and those who did not. Moreover, we were able to differentiate early- and late-onset $\mathrm{PE}$ by drawing ROC curves to compare the two methods of screening for early-onset PE (Figure 2) and lateonset PE (Figure 3). By comparing the areas under the curves, it was shown that uterine Doppler mPI $(\mathrm{AUC}=0.90$, 95\% CI $(0.85-0.96) P<.001)$ seemed to perform better than maternal history alone (AUC $=0.75,95 \%$ CI $(0.63-0.87)$ $P=.001)$ in detection of early-onset $\mathrm{PE}$, although there was no statistical difference when we compared both AUCs $(P=$ .076). The performance of uterine Doppler mPI assessment of risk $(\mathrm{AUC}=0.70,95 \% \mathrm{CI}(0.61-0.78) P<.001)$ was not statistically different from maternal history $(\mathrm{AUC}=0.77$, 95\% CI $(0.69-0.84, P<.001)$ for late-onset PE $(P=.254)$. The combination of the two methods did little to increase 
TABLE 2: Screening characteristics for mean PI > 1.66 and/or bilateral Notches at $20 \mathrm{w}$ (screen positive rate: $9.2 \%$ ).

\begin{tabular}{|c|c|c|c|c|c|c|}
\hline & $\mathrm{S}(\%)$ & $\mathrm{E}(\%)$ & PPV (\%) & NPV (\%) & $\mathrm{LR}+$ & LR- \\
\hline $\mathrm{PE}$ & 48.0 & 91.3 & 6.5 & 99.3 & 5.5 & 0.18 \\
\hline $\mathrm{PE}<32 \mathrm{w}$ & 75.0 & 91.0 & 2.7 & 99.9 & 8.3 & 0.12 \\
\hline $\mathrm{PE} \geq 32 \mathrm{w}$ & 38.9 & 91.0 & 3.8 & 99.4 & 4.3 & 0.23 \\
\hline IUGR & 52.2 & 91.3 & 6.5 & 99.4 & 6.0 & 0.17 \\
\hline IUGR $<32 \mathrm{w}$ & 72.7 & 91.1 & 4.3 & 99.8 & 8.2 & 0.12 \\
\hline IUGR $\geq 32 \mathrm{w}$ & 33.3 & 90.9 & 2.2 & 99.6 & 3.7 & 0.27 \\
\hline $\mathrm{PE}$ and/or IUGR $<32$ & 73.7 & 91.2 & 5.0 & 99.8 & 8.4 & 0.12 \\
\hline PE and IUGR & 65.2 & 91.0 & 2.7 & 99.9 & 7.2 & 0.14 \\
\hline $\mathrm{PE}$ and IUGR $<32 \mathrm{~s}$ & 73.3 & 90.9 & 2.0 & 99.9 & 8.1 & 0.12 \\
\hline $\mathrm{PE}$ and IUGR $\geq 32 \mathrm{~s}$ & 50.0 & 90.8 & 0.7 & 99.9 & 5.5 & 0.18 \\
\hline
\end{tabular}

TABle 3: Screening characteristics for mean PI > 1.40 irrespective of bilateral Notches at $20 \mathrm{w}$ (screen positive rate: 10\%).

\begin{tabular}{|c|c|c|c|c|c|c|}
\hline & $\mathrm{S}(\%)$ & $\mathrm{E}(\%)$ & PPV (\%) & NPV (\%) & $\mathrm{LR}+$ & LR- \\
\hline $\mathrm{PE}$ & 46.0 & 90.0 & 5.2 & 99.3 & 4.7 & 0.21 \\
\hline $\mathrm{PE}<32 \mathrm{~W}$ & 70.6 & 90.0 & 2.0 & 99.9 & 7.4 & 0.14 \\
\hline $\mathrm{PE} \geq 32 \mathrm{w}$ & 39.2 & 90.0 & 3.3 & 99.4 & 3.9 & 0.20 \\
\hline IUGR & 57.1 & 90.3 & 5.9 & 99.5 & 6.0 & 0.17 \\
\hline IUGR $<32 w$ & 73.3 & 90.1 & 3.6 & 99.9 & 7.4 & 0.13 \\
\hline IUGR $\geq 32 \mathrm{w}$ & 42.4 & 90.0 & 3.3 & 99.4 & 3.9 & 0.25 \\
\hline $\mathrm{PE}$ and/or IUGR $<32$ & 71.4 & 90.0 & 4.1 & 99.8 & 7.3 & 0.14 \\
\hline PE and IUGR & 68.4 & 90.0 & 2.1 & 99.9 & 6.8 & 0.15 \\
\hline $\mathrm{PE}$ and $\mathrm{IUGR}<32 \mathrm{~s}$ & 75.0 & 89.9 & 1.5 & 99.9 & 7.5 & 0.13 \\
\hline $\mathrm{PE}$ and IUGR $\geq 32 \mathrm{~s}$ & 57.1 & 89.9 & 0.7 & 99.9 & 5.6 & 0.18 \\
\hline
\end{tabular}

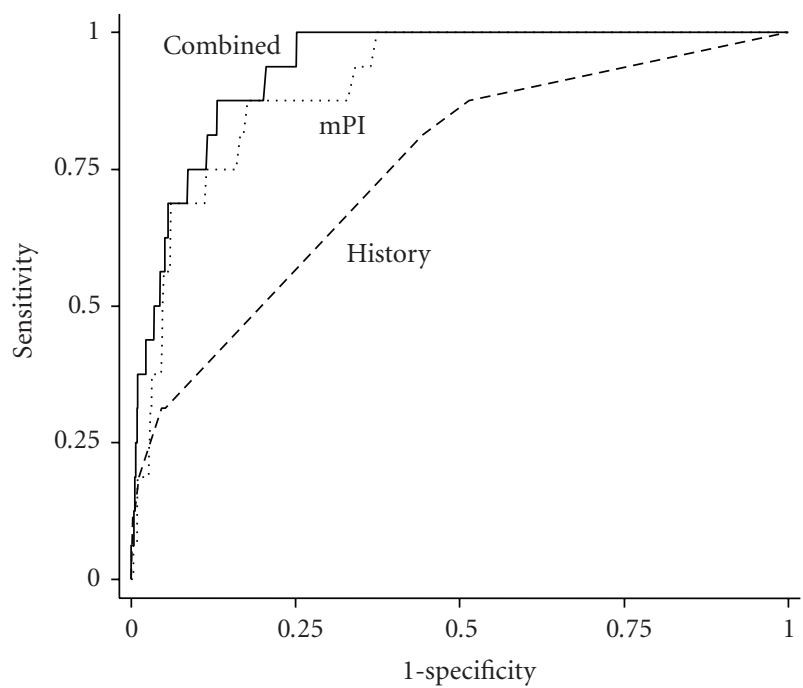

FIGURE 2: Receiver-operating characteristic curves showing the sensitivities for given screen-positive rates of uterine Doppler mean PI assessment (mPI), maternal history characteristics (History) and the combination of both methods (Combined) in the detection of early-onset PE.

the sensitivity of either early- or late-onset PE. Uterine artery Doppler performance for risk assessment of early-onset PE in the low-risk population $(\mathrm{AUC}=0.91,95 \% \mathrm{CI}(0.850-0.972)$

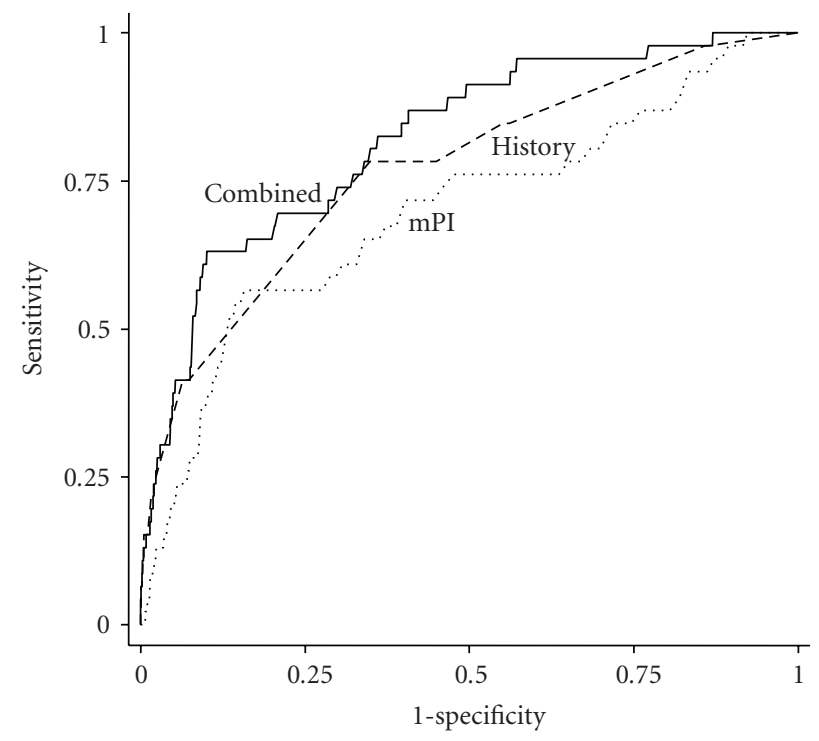

FIGURE 3: Receiver-operating characteristic curves showing the higher sensitivities for given screen-positive rates of uterine Doppler mean PI assessment (mPI), maternal history characteristics (History) and the combination of both methods (Combined) in the detection of late-onset PE.

$P<.002)$ and in the high-risk population (AUC $=0.87,95 \%$ CI (0.718-1.00) $P=.062)$ showed no differences $(P=.53)$ in detection of early-onset PE. 


\section{Discussion}

The results of this study demonstrate that uterine artery Doppler is an effective screening program for the prediction of PE and IUGR in an unselected population with classically low incidence of PE. Our findings have shown that measurement of the uterine artery mPI at 20 weeks gestation can identify $70.6 \%$ of pregnancies that will subsequently develop early-onset $\mathrm{PE}$ and $73.3 \%$ of pregnancies that develop early-onset IUGR, with a $10 \%$ false-positive rate.

In our screening program, the gestational age was moved to 19-22 weeks, which is when the routine abnormality scan is normally performed in most countries. Although a two-stage screening program has been recommended due to a higher false-positive rate around 20 weeks of gestation [21], we have found that, for the same false-positive rate, a one-stage screening test at 20 weeks gestation has a similar detection rate for PE or IUGR to studies performed at 22-24 weeks gestation [9].

We have also determined that with the same false-positive rate $(10 \%)$, uterine Doppler mPI gave similar results to bilateral notches and mPI, so the addition of bilateral notches did nothing to improve screening characteristics. Moreover, the use of the PI has removed any subjectivity associated with the definition and interpretation of the presence of notches in the waveform [7].

While there is no effective therapeutic measure for the prevention of PE and/or IUGR, NICE guideline recommendations point out that a woman's degree of risk for $\mathrm{PE}$ should be evaluated so that an appropriate plan for subsequent scheduling of antenatal appointments [22] and ultrasound growth scans can be formulated. There are no direct iatrogenic procedures that could affect mother or fetus after being classified as high risk for these conditions, so it would be reasonable to maximize the detection rate even at the cost of a slightly higher screen-positive rate. Nonetheless, such a classification may cause anxiety even though increased surveillance can improve maternal and neonatal outcome. We have found that uterine Doppler mPI had a good sensitivity even in the low-risk population, precisely, the group of patients that benefit most from a program of specific follow-up.

We have considered early-onset PE and IUGR as different outcomes from those conditions that were diagnosed near to term. Previous studies have established that uterine Doppler is much better at identifying the more severe early-onset cases [14, 23-26]. It has also been demonstrated that uterine Doppler screening is better in predicting severe early-onset disease or PE associated with IUGR, with sensitivities of 80 to 90\% [9]. To add to this, we have shown that uterine Doppler mPI performed better than maternal history in the detection of early-onset PE, whereas maternal history alone is similar to uterine Doppler mPI in the detection of late-onset PE. The combination of both methods did not significantly improve the sensitivity in early- or late-onset disease. We are aware of the limitations of maternal historical data as this piece of information relies on medical records and maternal selfreporting.
The classification of $\mathrm{PE}$ at or near term has clinical importance since early-onset PE is commonly associated with IUGR, abnormal uterine Doppler, and adverse maternal and neonatal outcomes [17, 27-29]. In contrast, late-onset $\mathrm{PE}$ is mainly associated with a milder form of maternal illness and low rate of fetal involvement, so perinatal outcome is usually favorable $[17,28]$. Patients with severe early-onset $\mathrm{PE}$ have different risk factors compared to late-onset PE $[29,30]$. Moreover, we have recently demonstrated that patients with early-onset PE ( $<32$ weeks) had abnormal uterine Doppler mPI, whereas, in late-onset PE ( $>32$ weeks), only a proportion of these cases presented abnormal uterine Doppler assessment, suggesting that there is a subgroup of late-onset cases with minimal placental involvement [28].

In conclusion, our results now show that a single 20 weeks uterine Doppler assessment was feasible and useful and is able to detect pregnant women with a high risk for early-onset adverse outcomes that relate to impaired trophoblast invasion in the low- and high-risk populations. In women with late-onset $\mathrm{PE}$, maternal history and uterine Doppler are less effective in identifying those women at risk, signifying that late-onset PE could result from heterogeneous causes in the context of absent or minimal placental impairment. These findings require a shift away from the general concept of PE as a single entity toward defining different types of PE classified according to cause. The application of this screening test in routine clinical practice needs future studies to demonstrate that uterine artery Doppler is useful to identify the population with a higher risk to pregnancy complications for whom intensive surveillance could improve maternal and fetal health.

\section{Acknowledgment}

We thank Bill Mulholland for help with the English version of the manuscript. Supported by grants from the Fondo de Investigación Sanitaria (FIS 01/1397, 02/0742, 06/1213 and 07/1095), and Centre Network RCMN (C03/08), and Group Network (G03/054), and Maternal and Child Network RETIC (RD 08/0072) financed by the Carlos III Institute of Health (Madrid, Spain).

\section{References}

[1] J. C. Hauth, M. G. Ewell, R. J. Levine, et al., "Pregnancy outcomes in healthy nulliparas who developed hypertension," Obstetrics \& Gynecology, vol. 95, no. 1, pp. 24-28, 2000.

[2] F. De Wolf, C. De Wolf-Peeters, I. Brosens, and W. B. Robertson, "The human placental bed: electron microscopic study of trophoblastic invasion of spiral arteries," American Journal of Obstetrics and Gynecology, vol. 137, no. 1, pp. 5870, 1980.

[3] J. W. Meekins, R. Pijnenborg, M. Hanssens, I. R. McFadyen, and A. van Asshe, "A study of placental bed spiral arteries and trophoblast invasion in normal and severe pre-eclamptic pregnancies," BJOG: An International Journal of Obstetrics \& Gynaecology, vol. 101, no. 8, pp. 669-674, 1994.

[4] S. Campbell, D. R. Griffen, J. M. Pearce, et al., "New Doppler technique for assessing uteroplacental blood flow," The Lancet, vol. 321, no. 8326, pp. 675-677, 1983. 
[5] A. T. Papageorghiou, C. K. H. Yu, and K. H. Nicolaides, "The role of uterine artery Doppler in predicting adverse pregnancy outcome," Best Practice and Research: Clinical Obstetrics and Gynaecology, vol. 18, no. 3, pp. 383-396, 2004.

[6] F. Prefumo, N. J. Sebire, and B. Thilaganathan, "Decreased endovascular trophoblast invasion in first trimester pregnancies with high-resistance uterine artery Doppler indices," Human Reproduction, vol. 19, no. 1, pp. 206-209, 2004.

[7] A. T. Papageorghiou, C. K. H. Yu, S. Cicero, S. Bower, and K. H. Nicolaides, "Second-trimester uterine artery Doppler screening in unselected populations: a review," Journal of Maternal-Fetal and Neonatal Medicine, vol. 12, no. 2, pp. 78$88,2002$.

[8] C. Lees, "Uterine artery Doppler: time to establish the ground rules," Ultrasound in Obstetrics and Gynecology, vol. 16, no. 7, pp. 607-609, 2000.

[9] A. T. Papageorghiou, C. K. H. Yu, R. Bindra, G. Pandis, and K. H. Nicolaides, "Multicenter screening for pre-eclampsia and fetal growth restriction by transvaginal uterine artery Doppler at 23 weeks of gestation," Ultrasound in Obstetrics and Gynecology, vol. 18, no. 5, pp. 441-449, 2001.

[10] S. A. Steel, J. M. Pearce, P. McParland, and G. V. P. Chamberlain, "Early Doppler ultrasound screening in prediction of hypertensive disorders of pregnancy," The Lancet, vol. 335, no. 8705, pp. 1548-1551, 1990.

[11] S. Bower, K. Schuchter, and S. Campbell, "Doppler ultrasound screening as part of routine antenatal scanning: prediction of pre-eclampsia and intrauterine growth retardation," British Journal of Obstetrics and Gynaecology, vol. 100, no. 11, pp. 989994, 1993.

[12] K. Harrington, D. Cooper, C. Lees, K. Hecher, and S. Campbell, "Doppler ultrasound of the uterine arteries: the importance of bilateral notching in the prediction of preeclampsia, placental abruption or delivery of a small-forgestational-age baby," Ultrasound in Obstetrics and Gynecology, vol. 7, no. 3, pp. 182-188, 1996.

[13] T. Frusca, M. Soregaroli, A. Valcamonico, F. Guandalini, and L. Danti, "Doppler velocimetry of the uterine arteries in nulliparous women," Early Human Development, vol. 48, no. 1-2, pp. 177-185, 1997.

[14] G. Albaiges, H. Missfelder-Lobos, C. Lees, M. Parra, and K. H. Nicolaides, "One-stage screening for pregnancy complications by color Doppler assessment of the uterine arteries at 23 weeks' gestation," Obstetrics \& Gynecology, vol. 96, no. 4, pp. 559-564, 2000 .

[15] W. Kurdi, S. Campbell, J. Aquilina, P. England, and K. Harrington, "The role of color Doppler imaging of the uterine arteries at 20 weeks' gestation in stratifying antenatal care," Ultrasound in Obstetrics and Gynecology, vol. 12, no. 5, pp. 339-345, 1998.

[16] J. Aquilina, O. Thompson, B. Thilaganathan, and K. Harrington, "Improved early prediction of pre-eclampsia by combining second-trimester maternal serum inhibin-A and uterine artery Doppler," Ultrasound in Obstetrics and Gynecology, vol. 17, no. 6, pp. 477-484, 2001.

[17] B. Sibai, G. Dekker, and M. Kupferminc, "Pre-eclampsia," The Lancet, vol. 365, no. 9461, pp. 785-799, 2005.

[18] F. Crispi, E. Llurba, C. Domínguez, P. Martín-Gallán, L. Cabero, and E. Gratacós, "Predictive value of angiogenic factors and uterine artery Doppler for early- versus lateonset pre-eclampsia and intrauterine growth restriction," Ultrasound in Obstetrics and Gynecology, vol. 31, no. 3, pp. 303-309, 2008.
[19] F. P. Zuspan, "The hypertensive disorders of pregnancy," Technical Report Series 758, WHO, Geneva, Switzerland, 1978.

[20] P. W. Soothill, C. S. Bobrow, and R. Holmes, "Small for gestational age is not a diagnosis," Ultrasound in Obstetrics and Gynecology, vol. 13, no. 4, pp. 225-228, 1999.

[21] S. Bower, K. Schuchter, and S. Campbell, "Doppler ultrasound screening as part of routine antenatal scanning: prediction of pre-eclampsia and intrauterine growth retardation," BJOG: An International Journal of Obstetrics \& Gynaecology, vol. 100, no. 11, pp. 989-994, 1993.

[22] The Guideline Development Group (GDG), 2008.

[23] A. T. Papageorghiou, C. K. H. Yu, I. E. Erasmus, H. S. Cuckle, and K. H. Nicolaides, "Assessment of risk for the development of pre-eclampsia by maternal characteristics and uterine artery Doppler," BJOG: An International Journal of Obstetrics \& Gynaecology, vol. 112, no. 6, pp. 703-709, 2005.

[24] K. Harrington, D. Cooper, C. Lees, K. Hecher, and S. Campbell, "Doppler ultrasound of the uterine arteries: the importance of bilateral notching in the prediction of preeclampsia, placental abruption or delivery of a small-forgestational-age baby," Ultrasound in Obstetrics and Gynecology, vol. 7, no. 3, pp. 182-188, 1996.

[25] W. Kurdi, S. Campbell, J. Aquilina, P. England, and K. Harrington, "The role of color Doppler imaging of the uterine arteries at 20 weeks' gestation in stratifying antenatal care," Ultrasound in Obstetrics and Gynecology, vol. 12, no. 5, pp. 339-345, 1998.

[26] A. T. Papageorghiou, C. K. H. Yu, R. Bindra, G. Pandis, and K. H. Nicolaides, "Multicenter screening for pre-eclampsia and fetal growth restriction by transvaginal uterine artery Doppler at 23 weeks of gestation," Ultrasound in Obstetrics and Gynecology, vol. 18, no. 5, pp. 441-449, 2001.

[27] D. J. Murphy and G. M. Stirrat, "Mortality and morbidity associated with early-onset pre-eclampsia," Hypertension in Pregnancy, vol. 19, no. 2, pp. 221-231, 2000.

[28] F. Crispi, C. Dominguez, E. Llurba, P. Martin-Gallan, L. Cabero, and E. Gratacós, "Placental angiogenic growth factors and uterine artery Doppler findings for characterization of different subsets in pre-eclampsia and in isolated intrauterine growth restriction," American Journal of Obstetrics and Gynecology, vol. 195, no. 1, pp. 201-207, 2006.

[29] R. B. Ness and B. M. Sibai, "Shared and disparate components of the pathophysiologies of fetal growth restriction and preeclampsia," American Journal of Obstetrics and Gynecology, vol. 195, no. 1, pp. 40-49, 2006.

[30] B. M. Sibai, "Maternal and uteroplacental hemodynamics for the classification and prediction of pre-eclampsia," Hypertension, vol. 52, no. 5, pp. 805-806, 2008. 


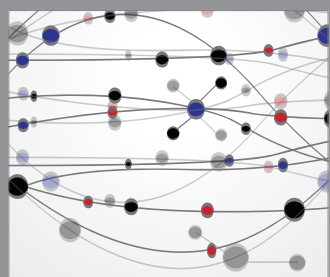

The Scientific World Journal
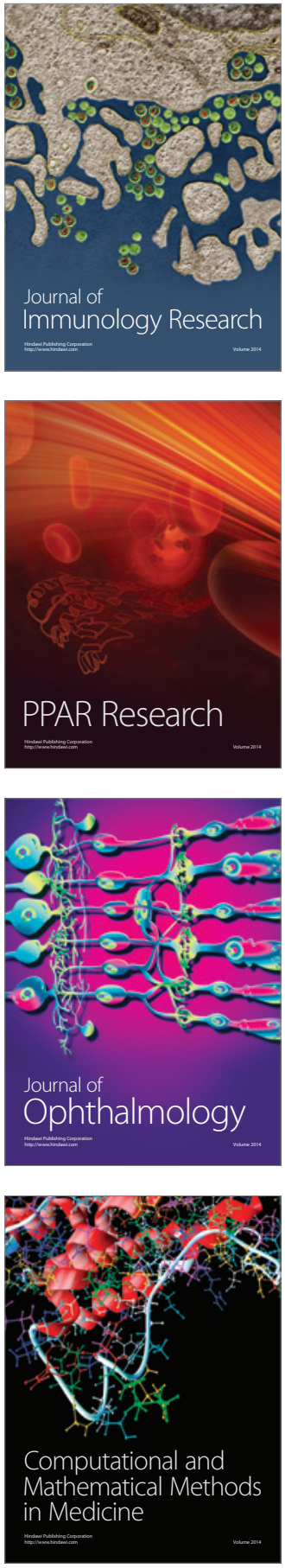

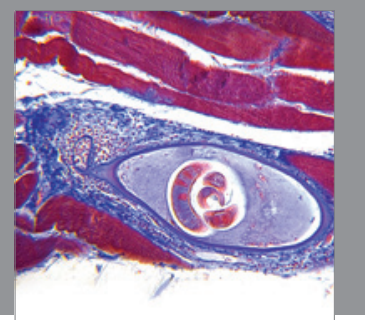

Gastroenterology

Research and Practice
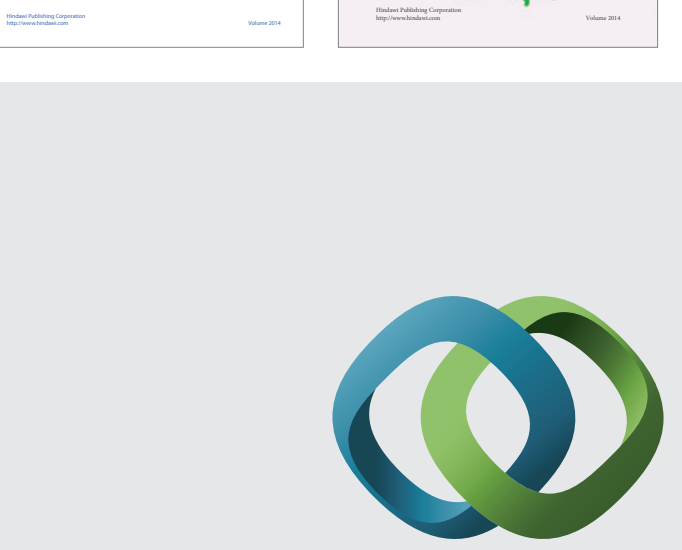

\section{Hindawi}

Submit your manuscripts at

http://www.hindawi.com
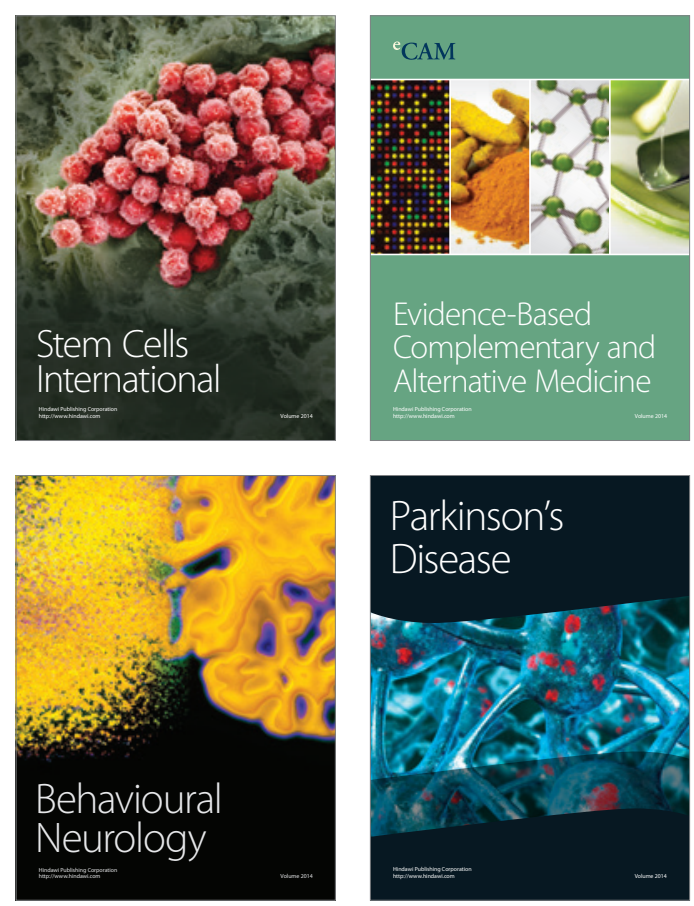

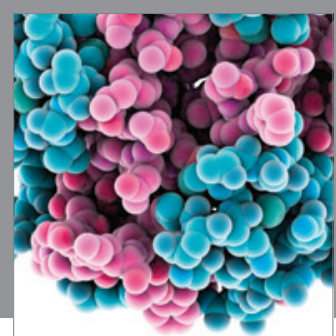

Journal of
Diabetes Research

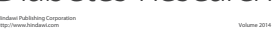

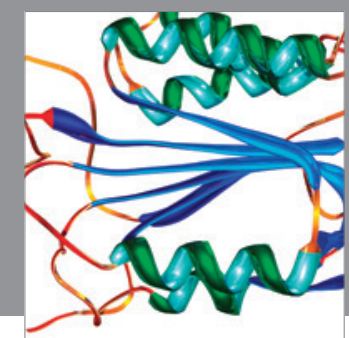

Disease Markers
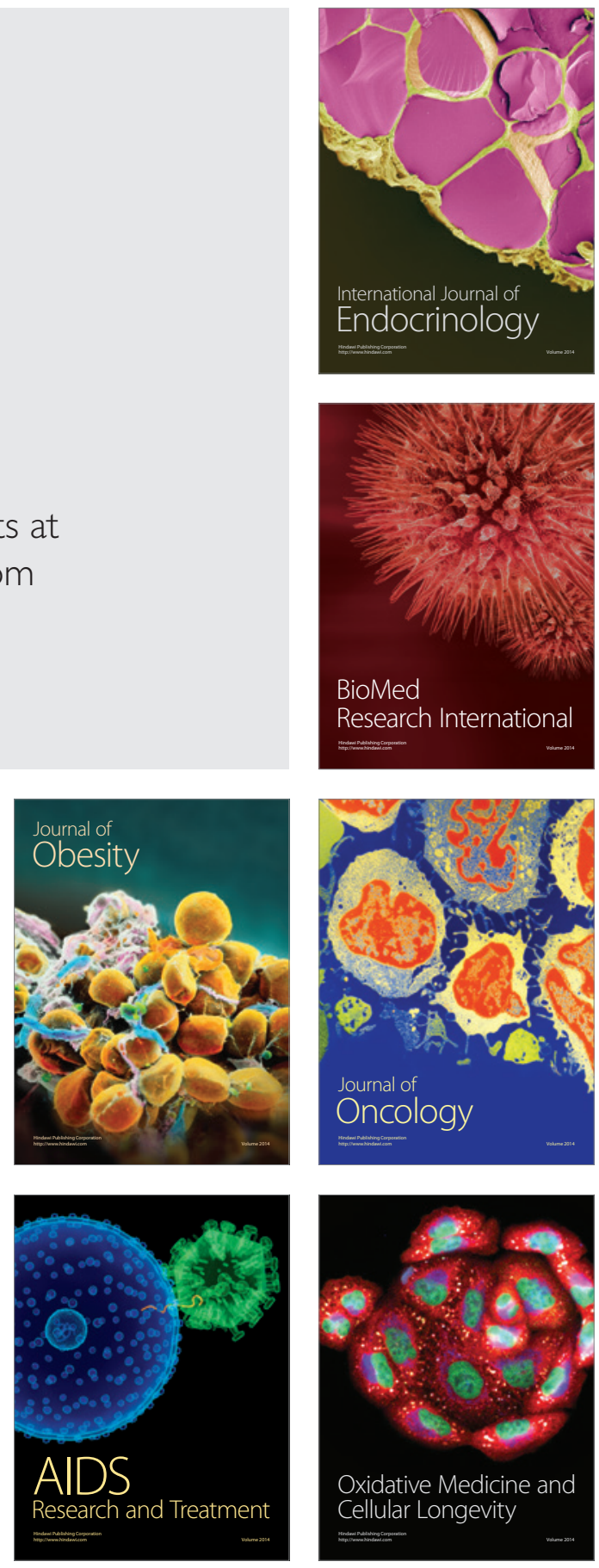